\title{
Antigenic Relationships among Bacteroides Species Studied by Rocket-Line Immunoelectrophoresis
}

\author{
FRANÇOISE TESSIER,* CLAUDINE QUENTIN, MICHÈLE CAPDEPUY, AND ROLAND GUINET† \\ Laboratoire de Microbiologie, U.F.R. des Sciences Pharmaceutiques, Université de Bordeaux II, \\ 146, rue Léo Saignat, 33076 Bordeaux Cedex, France
}

\begin{abstract}
Antigenic relationships among the 10 Bacteroides species, Porphyromonas gingivalis, Prevotella bivia, and Prevotella intermedia were investigated by rocket-line immunoelectrophoresis, using antigenic extracts prepared from type strains and antisera to Bacteroides fragilis, Bacteroides distasonis, Bacteroides thetaiotaomicron, Porphyromonas gingivalis, and Prevotella intermedia. Among the members of the genus Bacteroides, $B$. fragilis, Bacteroides caccae, Bacteroides stercoris, Bacteroides vulgatus, B. thetaiotaomicron, and Bacteroides ovatus were found to be closely related, and $B$. distasonis and Bacteroides merdae were the species that were most distantly related to $B$. fragilis; Bacteroides eggerthii and Bacteroides uniformis were in an intermediate position. Porphyromonas gingivalis, Prevotella bivia, and Prevotella intermedia shared very low percentages of common antigens with the Bacteroides species. Detection of enzymic activities in immunoprecipitates allowed us to identify some antigens to enzymes and to study the immunological similarities of the enzymes. The malate dehydrogenases of the Bacteroides species were genus specific, and the glucose-6-phosphate dehydrogenases of most species exhibited antigenic similarities. The esterases of $B$. distasonis and $B$. thetaiotaomicron appeared to be species specific, whereas the esterases and phosphatases of $B$. fragilis cross-reacted with the esterases and phosphatases of $B$. stercoris and $B$. vulgatus, as did the phosphatases of $B$. distasonis and $B$. merdae. The phosphatases of $B$. eggerthii, $B$. uniformis, Porphyromonas gingivalis, and Prevotella intermedia were antigenically similar.
\end{abstract}

Organisms belonging to the genus Bacteroides Castellani and Chalmers 1919 are obligately anaerobic, gram-negative, nonsporing, rod-shaped bacteria $(7,8)$. However, this genus is known to be phenotypically and phylogenetically extremely heterogeneous. Thus, the 39 species included in the genus in Bergey's Manual of Systematic Bacteriology (7) exhibit a variety of cellular morphologies and saccharolytic and proteolytic activities and have DNA base compositions ranging from 28 to $61 \mathrm{~mol} \%$ guanine plus cytosine.

On the basis of biochemical, chemical, and molecular biological criteria $(10,18,20)$, the majority of the more typical Bacteroides species form three broad groups. The first group consists of Bacteroides fragilis and related saccharolytic intestinal species. This group includes five species which at one time were described as subspecies of a single species, B. fragilis (8) (B. fragilis, Bacteroides vulgatus, Bacteroides ovatus, Bacteroides distasonis, and Bacteroides thetaiotaomicron), and five species which either have been recognized more recently or have been considered similar to B. fragilis (Bacteroides eggerthii, Bacteroides uniformis, Bacteroides caccae, Bacteroides merdae, and Bacteroides stercoris) $(10,11)$. The second group comprises a large number of moderately saccharolytic taxa, such as Bacteroides melaninogenicus and related species, including Bacteroides bivius and Bacteroides intermedius. The third group includes the asaccharolytic pigmented species Bacteroides asaccharolyticus, Bacteroides gingivalis, and Bacteroides endodontalis. Recently, Shah and Collins (20) have proposed that the genus Bacteroides should be restricted to the 10 species belonging to the $B$. fragilis group and that the members of the second and third groups should be transferred to the new genera Prevotella and Porphyromonas,

\footnotetext{
* Corresponding author.

$\dagger$ Present address: Centre d'Immunochimie Microbienne-Le Poirier, 69210 Lentilly, France.
}

respectively $(19,21)$. Several other new genera have been created (20) to accommodate many of the species formerly placed in the genus Bacteroides (7).

Previous immunological studies $(1,2,16)$ have demonstrated the antigenic complexity and heterogeneity of the genus Bacteroides. However, Rogemond and Guinet (17) have investigated the antigenic relationships among five species belonging to the $B$. fragilis group ( $B$. fragilis, $B$. distasonis, $B$. ovatus, $B$. thetaiotaomicron, and $B$. vulgatus) by using cross immunoelectrophoresis (CIE) and have shown that some of the antigens with enzymic activities are species or group specific. The purposes of this study were to further examine the antigenic relationships among the 10 species currently placed in the genus Bacteroides and to compare these species with species that are now excluded from the genus by using another immunochemical technique, rocket-line immunoelectrophoresis (RLIE) $(14,15)$.

\section{MATERIALS AND METHODS}

Strains. The 14 bacterial strains used in this study and their sources are shown in Table 1; 13 of the isolates are the type strains (7) of the 10 Bacteroides species, Porphyromonas gingivalis, Prevotella bivia, and Prevotella intermedia. $B$. fragilis IPL E323 was included as representative of serotype E2 since $B$. fragilis type strain NCTC 9343 belongs to serotype E1 according to the serological classification of Beerens et al. $(2,4)$.

Antigenic extracts. Strains were grown anaerobically for 72 $h$ at $37^{\circ} \mathrm{C}$ in TGY broth (Institut Pasteur Production) supplemented with a kanamycin-vancomycin mixture (BioMerieux, Craponne, France), vitamin $\mathrm{K}_{1}(0.1 \mu \mathrm{g} / \mathrm{ml})$, and hemin $(10 \mu \mathrm{g} / \mathrm{ml})$. Cells were harvested by centrifugation at $10,000 \times g$ for $15 \mathrm{~min}$. The resulting pellets were washed three times and resuspended in physiological saline. The cell suspensions were shaken for $2 \mathrm{~min}$ at $2,800 \mathrm{rpm}$ in a Braun homogeneizer by using 70-ml flasks (Braun Melsungen) filled 
TABLE 1. Cross-reactions among Bacteroides, Porphyromonas gingivalis, Prevotella bivia, and Prevotella intermedia antigens as determined by RLIE

\begin{tabular}{|c|c|c|c|c|c|c|}
\hline \multirow[b]{2}{*}{ Antigen } & \multirow[b]{2}{*}{$\begin{array}{l}\text { Protein } \\
\text { concn } \\
\text { (g/liter) }\end{array}$} & \multicolumn{5}{|c|}{$\%$ of common antigens with: } \\
\hline & & $\begin{array}{l}\text { B. fragilis } \\
\text { serotype } \\
\text { E1 } \\
\text { antiserum }\end{array}$ & $\begin{array}{l}\text { B. distasonis } \\
\text { antiserum }\end{array}$ & $\begin{array}{l}\text { B. thetaiotaomicron } \\
\text { antiserum }\end{array}$ & $\begin{array}{l}\text { Porphyromonas } \\
\text { gingivalis } \\
\text { antiserum }\end{array}$ & $\begin{array}{c}\text { Prevotella } \\
\text { intermedia } \\
\text { antiserum }\end{array}$ \\
\hline $\begin{array}{l}\text { B. fragilis serotype E1 strain } \\
\text { NCTC } 9343\end{array}$ & 8 & $100(19)^{a}$ & 53 & 83 & 3 & 21 \\
\hline $\begin{array}{l}\text { B. fragilis serotype E2 strain } \\
\text { IPL E323 }\end{array}$ & 12 & 100 & 53 & 83 & 3 & 21 \\
\hline B. caccae АТCC 43185 & 11 & 68 & 29 & 94 & 3 & 16 \\
\hline B. distasonis ATCC 8503 & 14 & 47 & $100(17)$ & 44 & 13 & 16 \\
\hline B. eggerthii ATCC 27754 & 16 & 47 & 35 & 44 & 7 & 11 \\
\hline B. merdae ATCC 43184 & 9 & 16 & 65 & 61 & 7 & 26 \\
\hline B. ovatus ATCC 8483 & 11 & 42 & 29 & 77 & 0 & 16 \\
\hline B. stercoris ATCC 43183 & 12 & 84 & 47 & 94 & 7 & 26 \\
\hline $\begin{array}{l}\text { B. thetaiotaomicron ATCC } \\
29148\end{array}$ & 12 & 47 & 29 & $100(18)$ & 0 & 21 \\
\hline B. uniformis ATCC 8492 & 8 & 53 & 41 & 67 & 7 & 21 \\
\hline B. vulgatus ATCC 8482 & 10 & 74 & 41 & 67 & 3 & 21 \\
\hline $\begin{array}{l}\text { Porphyromonas gingivalis } \\
\text { ATCC } 33277\end{array}$ & 12 & 26 & 41 & 22 & $100(30)$ & 11 \\
\hline Prevotella bivia ATCC 29303 & 10 & 5 & 0 & 11 & 0 & 26 \\
\hline $\begin{array}{l}\text { Prevotella intermedia ATCC } \\
25611\end{array}$ & 6 & 21 & 18 & 22 & 3 & $100(19)$ \\
\hline
\end{tabular}

${ }^{a}$ The numbers in parentheses are the numbers of rockets in the homologous systems.

with $50 \mathrm{~g}$ of fine beads (diameter, $0.5 \mathrm{~mm}$ ). The beads, cell debris, and unbroken cells were removed by centrifugation for $1 \mathrm{~h}$ at $20,000 \times g$, and the supernatants were freeze-dried and used for the study. Protein concentrations were estimated by the method of Lowry. Enzymes in antigenic extracts were detected either by zone electrophoresis in the first dimension (for glucose-6-phosphate dehydrogenase
[G6PDH] and malate dehydrogenase [MDH]) (17) or by the API-ZYM system (API System, La Balme les Grottes, France) (for alpha- and beta-esterases and phosphatase).

Antisera. Five polyclonal antisera were raised in rabbits against soluble extracts from $B$. fragilis serotype $\mathrm{E} 1, B$. distasonis, $B$. thetaiotaomicron, Porphyromonas gingivalis, and Prevotella intermedia. The rabbits were immunized by

TABLE 2. Enzymes detected by RLIE

\begin{tabular}{|c|c|c|c|c|c|c|c|c|c|c|c|c|c|c|c|c|c|c|c|c|}
\hline \multirow[b]{2}{*}{ Antigen } & \multicolumn{4}{|c|}{$\begin{array}{l}\text { B. fragilis serotype } \\
\text { E1 antiserum }\end{array}$} & \multicolumn{4}{|c|}{$\begin{array}{l}\text { B. distasonis } \\
\text { antiserum }\end{array}$} & \multicolumn{4}{|c|}{$\begin{array}{c}\text { B. thetaiotaomicron } \\
\text { antiserum }\end{array}$} & \multicolumn{4}{|c|}{$\begin{array}{c}\text { Porphyromonas } \\
\text { gingivalis antiserum }\end{array}$} & \multicolumn{4}{|c|}{$\begin{array}{c}\text { Prevotella intermedia } \\
\text { antiserum }\end{array}$} \\
\hline & 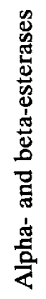 & 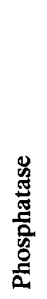 & 吾 & $\stackrel{\mathbf{T}}{\mathrm{z}}$ & 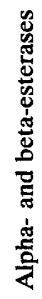 & 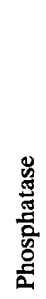 & 容 & $\stackrel{\text { 壱 }}{\mathrm{S}}$ & 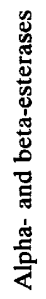 & 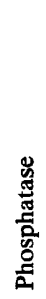 & $\underset{8}{8}$ & $\stackrel{\text { 突 }}{\mathrm{\Sigma}}$ & 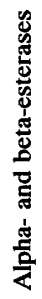 & 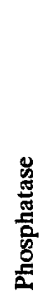 & $\begin{array}{l}\text { 采 } \\
\frac{8}{0}\end{array}$ & $\stackrel{\mathrm{I}}{\mathrm{S}}$ & 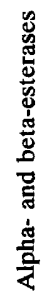 & 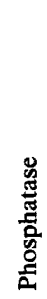 & $\frac{\text { I }}{2}$ & $\stackrel{\text { 帘 }}{\mathrm{S}}$ \\
\hline B. fragilis serotype E1 & + & + & + & + & - & - & - & + & - & - & + & - & - & - & - & - & - & - & - & - \\
\hline B. fragilis serotype E2 & + & + & + & + & - & - & - & + & - & - & + & - & - & - & - & - & - & - & - & - \\
\hline B. caccae & - & - & - & + & - & - & - & + & - & - & - & - & - & - & - & - & - & - & - & - \\
\hline B. distasonis & - & - & - & + & + & + & - & + & - & - & - & - & - & - & - & - & - & - & - & - \\
\hline B. eggerthii & - & - & + & + & - & - & - & + & - & - & + & - & - & + & - & - & - & + & - & - \\
\hline B. merdae & - & - & - & + & - & + & - & + & - & - & - & - & - & - & - & - & - & - & - & - \\
\hline B. ovatus & - & - & + & + & - & - & - & + & - & - & + & - & - & - & - & - & - & - & - & - \\
\hline B. stercoris & + & + & + & + & - & - & - & + & - & - & + & - & - & - & - & - & - & - & - & - \\
\hline B. thetaiotaomicron & - & - & + & + & - & - & - & + & + & - & + & - & - & - & - & - & - & - & - & - \\
\hline B. uniformis & - & - & + & + & - & - & - & + & - & - & + & - & - & + & - & - & - & + & - & - \\
\hline B. vulgatus & + & + & - & + & - & - & - & + & - & - & - & - & - & - & - & - & - & - & - & - \\
\hline Porphyromonas gingivalis & - & - & - & - & - & - & - & - & - & - & - & - & - & + & - & + & - & + & - & - \\
\hline Prevotella bivia & - & - & - & - & - & - & - & - & - & - & - & - & - & - & - & - & - & - & - & - \\
\hline Prevotella intermedia & - & - & - & - & - & - & - & - & - & - & - & - & - & + & - & - & + & + & - & - \\
\hline
\end{tabular}


using an antigen-muramyl dipeptide mixture (6), and the titers of serum antibodies were evaluated by line immunoelectrophoresis (13).

RLIE. The RLIE procedure used was essentially the procedure described by Kroll $(14,15)$; a $0.8 \%$ agarose gel (standard low- $M_{\mathrm{r}}$ gel; Bio-Rad) and $0.025 \mathrm{M}$ Tris buffer $(\mathrm{pH}$ 8.6) were used. Three gels were poured next to each other on GelBond sheets (Flobio, Courbevoie, France) from the cathode to the anode. One gel was $1.5 \mathrm{~mm}$ thick and contained holes (diameter, $2.5 \mathrm{~mm}$ ) that were $10 \mathrm{~mm}$ apart for the antigenic extracts that were to be compared; the second gel was $1.3 \mathrm{~mm}$ thick and contained the antigenic extract used as a reference; and the third gel was $1 \mathrm{~mm}$ thick and contained the antiserum that was homologous to the reference antigen. The optimal antigen-antibody ratio was determined in preliminary line immunoelectrophoresis experiments (6). The concentrations of antigens and antisera in the last two gels varied from 5 to $20 \%$ (vol/vol). RLIE was performed at $2 \mathrm{~V} / \mathrm{cm}$ for $18 \mathrm{~h}$. Unprecipitated proteins were removed by pressing the gels and washing them in buffered saline for $1 \mathrm{~h}$ and in distilled water for $2 \mathrm{~h}$. Then the gels were stained with Coomassie blue to detect all of the antigens and with various chromogenic substrates to detect the antigens with enzymic activities, as described previously (17). G6PDH, phosphatase, esterases, and MDH were detected successively. All of the antigenic extracts were tested against the different antisera at least 2 times and up to 12 times.

\section{RESULTS}

The cross-reactions among Bacteroides, Porphyromonas gingivalis, Prevotella bivia, and Prevotella intermedia antigens are shown in Fig. 1 and summarized in Table 1 . The protein concentrations of the soluble antigenic extracts varied from 6 to $16 \mathrm{~g} /$ liter, which is within the acceptable range for immunoelectrophoretic studies (17). In general, the rocket patterns were highly reproducible. The average number of rockets obtained in the homologous reactions varied from 17 to 30 . The numbers of rockets obtained in the heterologous reactions were counted, and the percentages of common antigens were evaluated by comparison with the corresponding homologous systems (defined as 100\%).

$\mathrm{MDH}$, esterases, and phosphatase were found in all antigenic extracts, whereas G6PDH was observed only in Bacteroides extracts. Cross-reactions among Bacteroides, Porphyromonas gingivalis, Prevotella bivia, and Prevotella

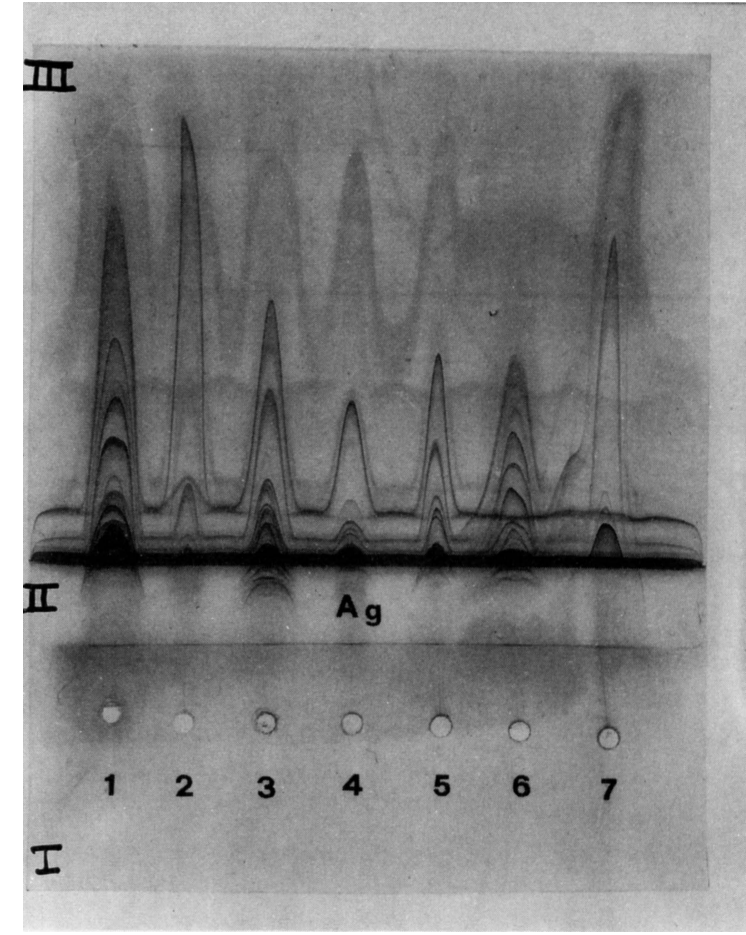

FIG. 1. RLIE pattern of $B$. thetaiotaomicron reference system obtained after Coomassie blue staining. Three gels were poured next to each other on GelBond sheets (Flobio) from the cathode to the anode. Gel I contained holes for the antigenic extracts to be compared; the wells were filled with $7-\mu$ l portions of soluble extracts from $B$. thetaiotaomicron (well 1), B. merdae (well 2), B. caccae (well 3), B. vulgatus (well 4), B. ovatus (well 5), B. fragilis E2 (well 6 ), and $B$. distasonis (well 7). Gel II contained the antigenic extract used as the reference extract $(\mathrm{Ag})(13 \mu \mathrm{l}$ of $B$. thetaiotaomicron soluble extract per $\mathrm{cm}^{2}$ of gel). Gel III contained the antiserum homologous to the reference antigen $(10 \mu \mathrm{l}$ of rabbit antiserum against $B$. thetaiotaomicron extract per $\mathrm{cm}^{2}$ ). After RLIE was performed, the rockets standing on precipitin lines expressed related antigens for the reference antigen and the other antigenic extracts.

intermedia antigens with enzymic activities are shown in Table 2 and Fig. 2. Particular enzymic activities were not detected in the precipitates obtained with four homologous systems; G6PDH was absent from $B$. distasonis, phos-

TABLE 3. Differentiation of the type strains of Bacteroides species on the basis of antigenic enzyme patterns determined by RLIE

\begin{tabular}{|c|c|c|c|c|c|c|}
\hline \multirow[b]{2}{*}{ Antigen } & \multicolumn{2}{|c|}{$\begin{array}{l}\text { B. fragilis serotype E1 } \\
\text { antiserum }\end{array}$} & \multicolumn{2}{|c|}{$\begin{array}{l}\text { B. distasonis } \\
\text { antiserum }\end{array}$} & \multirow{2}{*}{$\begin{array}{l}\text { B. thetaiotaomicron } \\
\text { antiserum: alpha- } \\
\text { and beta-esterases }\end{array}$} & \multirow{2}{*}{$\begin{array}{l}\text { Porphyromonas gingivalis } \\
\text { or Prevotella intermedia } \\
\text { antiserum: phosphatase }\end{array}$} \\
\hline & $\begin{array}{c}\text { Alpha- and } \\
\text { beta-esterases } \\
\text { or phosphatase }\end{array}$ & G6PDH & $\begin{array}{c}\text { Alpha- and } \\
\text { beta-esterases }\end{array}$ & Phosphatase & & \\
\hline B. fragilis & + & + & - & - & - & - \\
\hline B. caccae & - & - & - & - & - & - \\
\hline B. distasonis & - & - & + & + & - & - \\
\hline B. eggerthii & - & + & - & - & - & + \\
\hline B. merdae & - & - & - & + & - & - \\
\hline B. ovatus & - & + & - & - & - & - \\
\hline B. stercoris & + & + & - & - & - & - \\
\hline B. thetaiotaomicron & - & + & - & - & + & - \\
\hline B. uniformis & - & + & - & - & - & + \\
\hline B. vulgatus & + & - & - & - & - & - \\
\hline
\end{tabular}




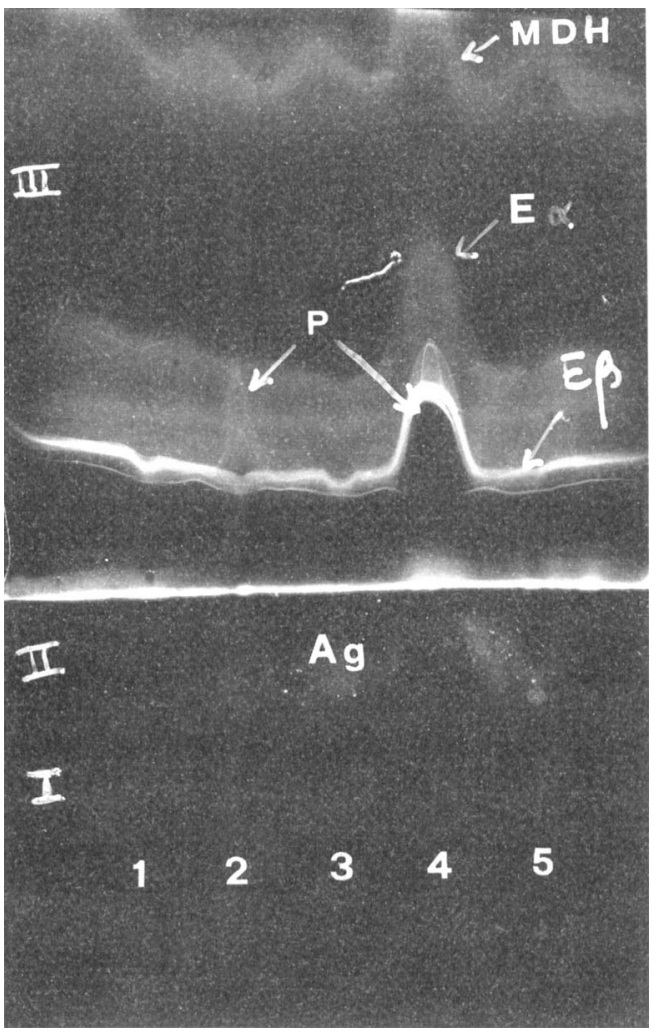

FIG. 2. Enzyme staining of the RLIE pattern of the $B$. distasonis reference system. Gel I contained wells that were filled with $7-\mu \mathrm{l}$ portions of soluble extracts from $B$. stercoris (well 1 ), B. merdae (well 2), B. fragilis E2 (well 3), B. distasonis (well 4), and B. caccae (well 5). Gel II contained the antigenic extract used as the reference extract $(\mathrm{Ag})\left(13 \mu \mathrm{l}\right.$ of $B$. distasonis soluble extract per $\mathrm{cm}^{2}$ of gel). Gel III contained the antiserum homologous to the reference antigen $\left(10 \mu \mathrm{l}\right.$ of rabbit antiserum against $B$. distasonis extract per $\left.\mathrm{cm}^{2}\right)$. After RLIE was performed, the gel was stained with various chromogenic substrates to detect phosphatase $(\mathrm{P})$, alpha-esterase $(E \alpha)$, beta-esterase $(E \beta)$, and MDH enzymic activities, giving specifically colored precipitin lines. The rockets standing on the precipitin lines expressed related antigens within the enzymic activities. Alpha-esterase and beta-esterase of $B$. distasonis are species-specific antigens since the corresponding rockets are present only in front of well 4 . Two rockets derived from $B$. merdae (well 2 ) and $B$. distasonis (well 4) are present on the precipitate line corresponding to phosphatase, suggesting that the two antigens are related. Similarly, MDH rockets are present in front of all of the soluble extracts tested, indicating that the corresponding antigens are identical.

phatase and MDH were absent from $B$. thetaiotaomicron, esterases were absent from Porphyromonas gingivalis, and MDH was absent from Prevotella intermedia.

Table 3 shows the differentiation of the type strains of the 10 Bacteroides species on the basis of three enzymic activities (esterases, phosphatase, and G6PDH) and four antisera (anti- $B$. fragilis, anti- $B$. distasonis, anti- $B$. thetaiotaomicron, and anti-Porphyromonas gingivalis or anti-Prevotella intermedia antisera).

\section{DISCUSSION}

Because of the diversity of the organisms included in the genus Bacteroides, many techniques have been used to clarify the taxonomy of the genus. Knivett et al. (12) have established a numerical classification of the genus Bacte- roides based on physiological properties, and Johnson (9) and Johnson and Harich (10) have categorized these organisms according to their levels of nucleic acid homology. Rogemond and Guinet (17) have compared the antigens and enzymes of $B$. fragilis, $B$. distasonis, $B$. ovatus, $B$. thetaiotaomicron, and $B$. vulgatus by using CIE and $B$. fragilis and B. distasonis antisera.

In this study, we investigated the antigenic relationships among the 10 Bacteroides species, Porphyromonas gingivalis, Prevotella bivia, and Prevotella intermedia by using $B$. fragilis, $B$. distasonis, $B$. thetaiotaomicron, Porphyromonas gingivalis, and Prevotella intermedia antisera. RLIE allowed us to compare more antigenic extracts on one slide than CIE. With regard to the total antigens, RLIE performed with the homologous systems verified that the Bacteroides, Porphyromonas, and Prevotella species are antigenically complex, since 17 to 30 rockets were detected. However, still more antigenic precipitates were identified for Bacteroides spp. by CIE (17). The results of RLIE performed with the heterologous systems confirmed the antigenic similarities and dissimilarities among species. The two $B$. fragilis strains used (serotype E1 and E2 strains) were found to be $100 \%$ similar and gave identical percentages of common antigens with all antisera. The RLIE patterns of $B$. fragilis, $B$. caccae, B. stercoris, B. vulgatus, B. thetaiotaomicron, and $B$. ovatus were very similar, and $B$. distasonis and $B$. merdae appeared to be the species that are most distantly related to $B$. fragilis; B. eggerthii and B. uniformis were in an intermediate position. The percentages of common antigens detected by RLIE and CIE (17) for the same pairs of Bacteroides extracts and antisera were somewhat different, probably because of differences in the techniques and antigen or antiserum titers used. The strains of Porphyromonas gingivalis, Prevotella bivia, and Prevotella intermedia tested exhibited very low percentages of common antigens with each other and, as expected, with the Bacteroides species.

Previously published data $(9,10,12,17)$ and data from this study support the existence of at least the following three major clusters in the genus Bacteroides: (i) $B$. fragilis and $B$. thetaiotaomicron; (ii) $B$. eggerthii and $B$. uniformis; and (iii) $B$. distasonis. When $B$. caccae was studied (10; this paper), it was found to be similar to $B$. fragilis, and $B$. merdae was found to be similar to $B$. distasonis. The taxonomic position of $B$. ovatus and $B$. vulgatus is variable, depending on the authors. $B$. ovatus and $B$. vulgatus had more antigens in common with $B$. fragilis in RLIE gels than in CIE gels. In contrast to the data of Johnson and Harich (10), we found that $B$. stercoris was more closely related to $B$. fragilis than to the $B$. eggerthii-B. uniformis cluster. Our results confirm that the group formerly designated the $B$. fragilis group is a homogeneous collection of distinct species (7) that includes, among others, the three recently recognized taxa $B$. caccae, $B$. merdae, and $B$. stercoris $(10,11)$. Our findings support the proposals that the genus Bacteroides should be confined to these organisms (20) and that Porphyromonas gingivalis (19), Prevotella bivia, and Prevotella intermedia (21) should be placed in separate genera.

The presence and absence of key enzymes are characteristics that have value in microbial classification. Shah and Collins $(18,21)$ have shown that Bacteroides spp. differ from Porphyromonas and Prevotella spp. by possessing enzymes of the pentose phosphate pathway, such as G6PDH, whereas all three genera have MDH activity. All of the species tested in this study produce esterases and phosphatase, as revealed by the API-ZYM system. The presence and absence of 
enzymes in our antigenic extracts were in complete agreement with the data of Shah and Collins $(18,21)$.

Immunoenzymic patterns, which consist of a few easily characterized precipitates, could be useful identification tools (17). However, as previously observed (17), although some enzymic activities are present in antigenic extracts, they could not be detected in the precipitates obtained with homologous systems. The reason for these discrepancies could be either a lack of the corresponding antibodies in antisera or the neutralizing properties of the antibodies. Whatever the cause, negative reactions cannot be interpreted when homologous reactions are unavailable or negative.

According to our results and as far as we could detect, the MDH of the Bacteroides species were all antigenically similar and quite different from the MDH of Porphyromonas gingivalis. The G6PDH of $B$. fragilis, $B$. thetaiotaomicron, $B$. eggerthii, B. uniformis, B. ovatus, and B. stercoris exhibited antigenic similarities. The esterases of $B$. distasonis, $B$. thetaiotaomicron, and Prevotella intermedia appeared to be species specific. The esterases and phosphatases of $B$. fragilis, $B$. stercoris, and $B$. vulgatus exhibited antigenic similarities, as did the phosphatases of $B$. distasonis and $B$. merdae. The phosphatases of $B$. eggerthii, B. uniformis, Porphyromonas gingivalis, and Prevotella intermedia were antigenically similar. Most of the results obtained by RLIE were consistent with the CIE results (17); the exceptions were the results for the esterases and/or the phosphatases of $B$. ovatus and $B$. vulgatus.

Bacteroides species are phenotypically very similar, and some of them are important human pathogens (5). Therefore, an identification scheme for these organisms based on simplified immunoenzymic patterns would be helpful, as suggested by Rogemond and Guinet (17). However, in our study, despite the fact that we used a higher number of antigenic extracts and antisera, it was still impossible to differentiate $B$. stercoris from $B$. fragilis and $B$. eggerthii from $B$. uniformis.

The use of monoclonal antisera to all of the species tested and the use of antigenic extracts from a larger set of strains should be helpful in further determining the antigenic relationships among Bacteroides, Porphyromonas, and Prevotella species.

\section{REFERENCES}

1. Babb, J. L., and C. S. Cummins. 1981. Relationships between serological groups and deoxyribonucleic acid homology groups in Bacteroides fragilis and related species. J. Clin. Microbiol. 13:369-379.

2. Beerens, H., P. Wattre, T. Shinjo, and C. Romond. 1971. Premiers résultats d'un essai de classification sérologique de 131 souches de Bacteroides du groupe fragilis. Ann. Inst. Pasteur (Paris) 121:187-198.

3. Cato, E. P., and J. L. Johnson. 1976. Reinstatement of species rank for Bacteroides fragilis, Bacteroides ovatus, Bacteroides distasonis, Bacteroides thetaiotaomicron, and Bacteroides vulgatus: designation of neotype strains for Bacteroides fragilis (Veillon and Zuber) Castellani and Chalmers and Bacteroides thetaiotaomicron (Distaso) Castellani and Zuber. Int. J. Syst. Bacteriol. 26:230-237.

4. Cherniak, R., G. L. Lombard, and V. R. Dowell, Jr. 1979.
Immunochemical evidence for multiple serotypes of Bacteroides fragilis. J. Clin. Microbiol. 9:699-704.

5. Duerden, B. I. 1983. The Bacteroidaceae: Bacteroides, Fusobacterium, and Leptotrichia, p. 114-136. In G. S. Wilson, A. A. Miles, and M. T. Parker (ed.), Topley and Wilson's principles of bacteriology, virology and immunity, 7th ed. Edward Arnold, London.

6. Gabriel-Bruneau, S. M., and R. M. F. Guinet. 1984. Antigenic relationships among some Candida species studied by crossedline immunoelectrophoresis: taxonomic significance. Int. J. Syst. Bacteriol. 34:227-236.

7. Holdeman, L. V., R. W. Kelley, and W. E. C. Moore. 1984. Genus I. Bacteroides Castellani and Chalmers 1919, p. 604-631. In N. R. Krieg and J. G. Holt (ed.), Bergey's manual of systematic bacteriology, vol. 1. The Williams \& Wilkins Co., Baltimore.

8. Holdeman, L. V., and W. E. C. Moore. 1974. Genus I. Bacteroides Castellani and Chalmers 1919, p. 385-404. In R. E. Buchanan and N. E. Gibbons (ed.), Bergey's manual of determinative bacteriology, 8th ed. The Williams \& Wilkins Co., Baltimore.

9. Johnson, J. L. 1978. Taxonomy of the Bacteroides. I. DNA homologies among Bacteroides fragilis and other saccharolytic Bacteroides species. Int. J. Syst. Bacteriol. 28:245-256.

10. Johnson, J. L., and B. Harich. 1986. Ribosomal ribonucleic acid homology among species of the genus Bacteroides. Int. J. Syst. Bacteriol. 36: 71-79.

11. Johnson, J. L., W. E. C. Moore, and L. V. H. Moore. 1986. Bacteroides caccae sp. nov., Bacteroides merdae sp. nov., and Bacteroides stercoris sp. nov. isolated from human feces. Int. J. Syst. Bacteriol. 36:499-501.

12. Knivett, V. A., H. N. Shah, A. S. McKee, and J. M. Hardie. 1983. Numerical taxonomy of some non-saccharolytic and saccharolytic Bacteroides species. J. Appl. Bacteriol. 55:71-80.

13. Kroll, J. 1973. Line immunoelectrophoresis, p. 61-67. In N. H. Axelsen, J. Kroll, and B. Weeke (ed.), A manual of quantitative immunoelectrophoresis. Methods and application. Blackwell Scientific Publications, Oslo.

14. Kroll, J. 1973. Rocket-line immunoelectrophoresis, p. 83-87. In N. H. Axelsen, J. Kroll, and B. Weeke (ed.), A manual of quantitative immunoelectrophoresis. Methods and application. Blackwell Scientific Publications, Oslo.

15. Kroll, J. 1983. Rocket-line immunoelectrophoresis. Scand. J. Immunol. Suppl. 10:165-169.

16. Lambe, D. W. 1980. Serology of Bacteroidaceae, p. 141-153. In D. W. Lambe, R. J. Genco, and K. J. Mayberry-Carson (ed.), Anaerobic bacteria. Selected topics. Plenum Press, New York.

17. Rogemond, V., and R. M. F. Guinet. 1985. Antigens and enzymes of Bacteroides of the Bacteroides fragilis group compared by crossed immunoelectrophoresis. Int. J. Syst. Bacteriol. 35:327-332.

18. Shah, H. N., and M. D. Collins. 1983. A review: genus Bacteroides: a chemotaxonomical perspective. J. Appl. Bacteriol. 55:403-416.

19. Shah, H. N., and M. D. Collins. 1988. Proposal for reclassification of Bacteroides asaccharolyticus, Bacteroides gingivalis, and Bacteroides endodontalis in a new genus, Porphyromonas. Int. J. Syst. Bacteriol. 38:128-131.

20. Shah, H. N., and M. D. Collins. 1989. Proposal to restrict the genus Bacteroides (Castellani and Chalmers) to Bacteroides fragilis and closely related species. Int. J. Syst. Bacteriol. 39:85-87.

21. Shah, H. N., and M. D. Collins. 1990. Prevotella, a new genus to include Bacteroides melaninogenicus and related species formerly classified in the genus Bacteroides. Int. J. Syst. Bacteriol. 40:205-208. 